\title{
Causative Factors for Femoral Pin Track Fractures in Navigated Total Knee Arthroplasty
}

\author{
TW Ewe, FRCS, EK Chee, MS Ortho, YS Chooi, MS Ortho, WM Ng*, MS Ortho
}

Department of Orthopaedics and Traumatology, Kuala Lumpur Hospital, Kuala Lumpur, Malaysia

*Department of Orthopaedic Surgery, University Malaya Medical Centre, Kuala Lumpur, Malaysia

\begin{abstract}
This retrospective radiographic analysis of 57 patients $(62$ knees) examined two possible factors involved in pin tract fractures of the femur due to navigated total knee arthroplasty (TKA): the angle of the tracker pin with respect to the lateral femoral cortex, and the distance between the tracker pin and the lateral joint line. Our findings demonstrate a relationship between postoperative pin tract induced stress fractures (3 patients), with pin tract angles exceeding $15^{\circ}$. Pin placement at a site more than $10 \mathrm{~cm}$ from the lateral joint line, did not show any significant association with risk of fracture. These findings lead to enhanced understanding of the causative factors underlying pin track femoral fractures in TKAs.
\end{abstract}

\section{Key Words:}

Computer Assisted Navigation, Total Knee Arthroplasty, Stress Fracture

\section{INTRODUCTION}

Computer assisted navigation in TKA has been gaining popularity due to validation of its increased accuracy for implantation of femoral and tibial components ${ }^{1-3}$. Most navigation systems require femoral and tibial pin insertion for placement of trackers. Unfortunately, the resultant bony defects act as stressors. Stress fractures involving previous pin site tracts in computer assisted TKAs have been reported with increasing frequency and various reasons have been put forward regarding the cause(s) of these fractures, without any real consensus ${ }^{48}$. The purpose of this study was to identify and analyse possible factors contributing to pin tract related fractures of the femur.

\section{MATERIALS AND METHODS}

We retrospectively reviewed the radiographs of consecutive patients who underwent computer assisted posterior stabilized primary total knee replacements (Scorpio NRG, Stryker) in our institution from January 2007 to December 2007. All the subject surgeries were performed by two of the institution's senior arthroplasty surgeons with the same protocol of tracker pin insertion utilized in each instance.

The placements of the 2 femur anchoring pins were made via separate, percutaneous stab incisions. These $3.0 \mathrm{~mm}$ threaded pins are self-drilling and self-tapping. We use the same instrument model (Stryker) in all cases, a wall mounted pneumatic drill system. The first (distal) pin is inserted bicortically in a medial to lateral direction, at a distance of approximately two finger breadths above the superior pole of the patella, angled 45 degrees to the sagittal plane of the femur. The jig for the tracker (OrthoLock, Stryker) is then placed over the distal pin. The second hole in the jig, which lies $2.5 \mathrm{~cm}$ proximal to the first, guides the insertion of the second pin, i.e., bicortically, at 30 degrees to the sagittal plane of the femur. Tibial pins are placed in a similar fashion in the anteromedial tibial diaphysis at a distance of four fingerbreadths, distal to the tibial tubercle. The tibial proximal pin is inserted first, followed by the distal pin in a similar fashion to the femur. A maximum of 2 attempts of pin placement are allow for each pin placement. The tracker is then attached, anatomical landmarks are recorded, and the TKA procedure is then completed. At the end of the procedure, all pins are removed with a power driver. Postoperatively, continuous passive motion exercises are started at Day 2. Full weight bearing is allowed on Day 3 with physiotherapist supervision. Subsequent follow-ups evaluations are conducted at fixed intervals, i.e., 2 weeks, 1 month, 3 months, and 6 months. Postoperatively, all patients achieved coronal varus and valgus angle of $\pm 3^{\circ}$

All radiographs were reviewed by two independent researchers. Two possible causative factors for fracture(s) were analysed (1) The angle made by the pin tract, with the lateral wall of the femur in the AP radiograph was analysed as to the oblique nature of the distal femoral pin as it penetrates the cortex. (2) The distance from the distal femoral pin to the lateral joint line was analysed (Figure 1).

Statistical analysis was performed utilizing SPSS (Version 14 for Windows), data were analysed using Chi Square test, a $p$ value of 0.05 was chosen. 


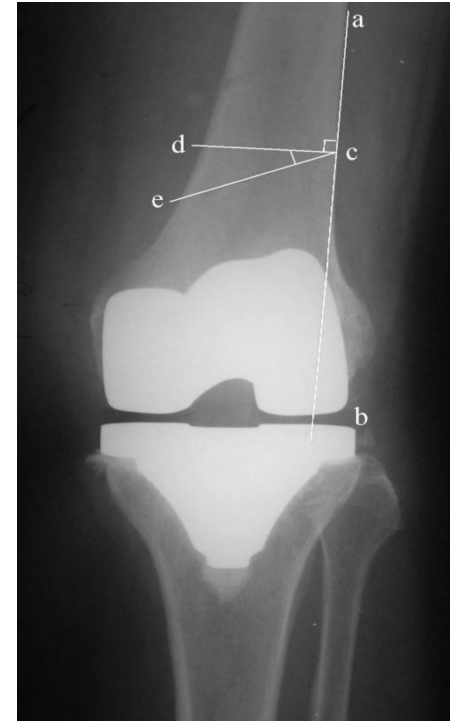

Fig. 1: Pin insertion angle is the angle made between the lines $C D$ and $C D$. $A B$ is the line drawn along lateral cortex of the femur. $C D$ is the line perpendicular to $A B$. $C E$ is the line connecting the two points made by the distal pin tract.
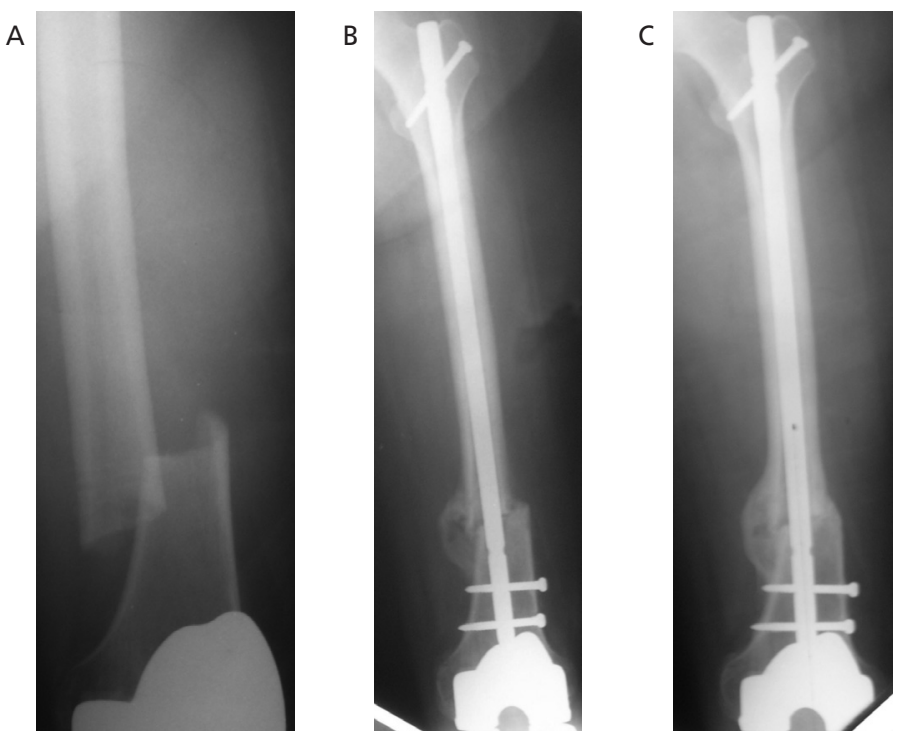

Fig. 2: (A) Fracture at the distal pin tract, 7-weeks after a navigated TKA. (B) 6 weeks after an interlocking nail for a pin-tract related fracture. (C) 6 months after an interlocking nail, showing abundant callus formation.

(Russel Taylor nail, Smith \& Nephew, Memphis). Bony union was achieved 4 months post-operatively.

Case 2. A 64 year old woman with bilateral knee osteoarthritis presented with a displaced fracture of the right femur, at the distal pin tract site, $11.6 \mathrm{~cm}$ from the lateral joint line 7 weeks post-operatively. The pin tract angle was $15^{\circ}$. An interlocking nail (Russel Taylor nail, Smith \& Nephew, Memphis) procedure was performed, with union achieved after 6 months. (Figure 2 A, B, C)

Case 3. This patient, presented with persistent left thigh pain postoperatively. Serial x-rays were unremarkable until 6 months post-operatively, when a fracture line appeared at the previous distal femur pin tract site, which was $7.5 \mathrm{~cm}$ from the lateral joint line. There was periosteal reaction. The pin tract angle was $22^{\circ}$. The patient was treated nonoperatively, i.e., protected weight-bearing, until bony union was achieved 3 months following diagnosis of the fracture. a distance of $10 \mathrm{~cm}$ or more from the distal femoral of to the lateral joint line, and of these only 2 knees had fractures; the remaining 31 knees with distance of less than $10 \mathrm{~cm}$ from the distal femoral pin to the lateral joint line, only 1 had a fracture. There was no significant association of fracture when the pin placement distance exceeded $10 \mathrm{~cm}$ from the lateral joint line.

Case 1. A 67year old woman with severe osteoarthritis of the left knee presented with a spontaneous fracture at the distal pin tract site, $13.2 \mathrm{~cm}$ from the lateral joint, 4 weeks postoperatively. The pin tract insertion angle was $20^{\circ}$. The fracture was subsequently stabilized with an interlocking nail

\section{DISCUSSION}

Numerous published studies cite cortical defects acting as stressors in the bone, but fractures associated with computerassisted navigation pins are rare. There is no single recommendation regarding the site for femur tracker pins. Various techniques include placing the pins at the metaphyseal / diaphyseal junction, $10 \mathrm{~cm}$ proximal to the knee joint, or into the femoral diaphysis, as far proximal as possible ${ }^{9,10}$. Although rare, this complication represents 
significant morbidity. A displaced fracture requiring operative fixation may negatively affect overall alignment, indirectly affecting implant survival and final outcome of the TKA. The duration required for fracture healing also disrupts the rehabilitation process. There is also the financial burden of additional surgery and hospitalisation secondary to these fractures.

Various causes have been postulated for these types of fractures. Ossendorf et al suggested that repeated attempts at pin placements increase the risk of stress fracture. $\mathrm{He}$ advocated unicortical pins, placed in an orthogonal fashion to avoid stress fractures ${ }^{1}$. Jung et al believed that these stress fracture are due to a misplaced fixation pin placed transcortically $^{2}$. Li et al found that 3 bicortically drilled holes decreases the strength of the femoral structure. Instead, he recommended small diameter anchoring pins or screws, and advised avoidance of eccentric drilling, especially in an overweight patients.

We postulate that biomechanically if an oblique pin tract is made (angle $\geq 15^{\circ}$ ), the tract may travel or involve longer distances of bone as compared to transverse insertion, thus causing increased stress in the bone and predisposing to fracture. We currently insert the pin as perpendicular as possible to the cortex and we also advocate bicortical distal pin placement, at the mainly cancellous site of the supracondylar metaphyseal region of the distal femur, at an angle of 90 degrees to the sagittal plane of the femur. We prefer unicortical pins for the second proximal pin as a safety precaution for the femur and tibia. Placement of the pins from a medial to lateral direction at about a 90-degree angle to the sagittal plane also avoids breaching the anterior cortex of the femur, which represents the tension side of the femur.

Limitations of this study include small sample size, and the fact that we investigated only two of several possible factors that may contribute to the risk of fracture (such as: osteoporosis, gender, obesity, height, weight and underlying systemic illness). Although of the number of cases reported herein is not large, we note that since applying these recommendations in our clinical orthopaedic practice, there have not been any pin tract related stress fractures in the subsequent 200 cases performed.

\section{CONCLUSION}

Navigated TKAs are not without complications. The incidence of fracture at pin insertion sites can be reduced significantly if we understand the contributing factors. There is association of femur fracture when insertion of the distal femoral pin angle exceeds a $15^{\circ}$ angle (from the lateral wall of the femur). However this problem does not seem to be affected by distance from the joint line. 


\section{REFERENCES}

1. Bathis H, Perlick L, Tingart M, Luring C, Zurakowski D, Grifka J. Alignment in total knee arthroplasty. A comparison of computer assisted surgery with the conventional technique. J Bone Joint Surg Br. 2004; 86: 682-7.

2. Decking R, Markmann Y, Fuchs J, Puhl W, Scharf HP. Leg axis after computer-navigated total knee arthroplasty: a prospective randomized trial comparing computer-navigated and manual implantation. J Arthroplasty, 2005; 20: 282-8.

3. Haaker RG, Stockheim M, Kamp M, Proff G, Breitenfelder J, Ottersbach A. Computer-assisted navigation increases precision of component placement in total knee arthroplasty. Clin Orthop Relat Res. 2005; 433: 152-9.

4. Ossendorf C, Fuchs B, Koch P. Femoral stress fracture after computer navigated total knee arthroplasty. Knee 2006; 13: 397-9.

5. Jung HJ, Jung YB, Song KS, Park SJ, Lee JS. Fracture associated with computer - navigated total knee arthroplasty: A report of two cases. J Bone Joint Surg Am. 2007; 89: 2280-4.

6. Li CH, Chen TH, Su YP, Shao PC, Lee KS, Chen WM. Periprosthetic femoral supracondylar fracture after total knee arthroplasty with navigation system. J Arthroplasty. 2008; 23: 304-7.

7. Robert W,Wysocki, Mitchell BS, Walter WV, Craig JDV. Femoral fracture through a previous pin site after computer - assisted total knee arthroplasty. J Arthroplasty. 2008: 23: 462-5.

8. Bonutti P, Dethmers D, Stiehl JB. Case report: femoral shaft fracture resulting from femoral tracker placement in navigated TKA. Clin Orthop Relat Res. 2008; 466(6): 1499-1502.

9. Stulberg SD, Loan P, Sarin V. Computer-assisted navigation in total knee replacement: results of an initial experience in thirtyfive patients. J Bone Joint Surg Am. 2002; 84-A(Suppl 2): 90.

10. Victor J, Hoste D. Image-based computer-assisted total knee arthroplasty leads to lower variability in coronal alignment. Clin Orthop Relat Res. 2004; 428: 131. 